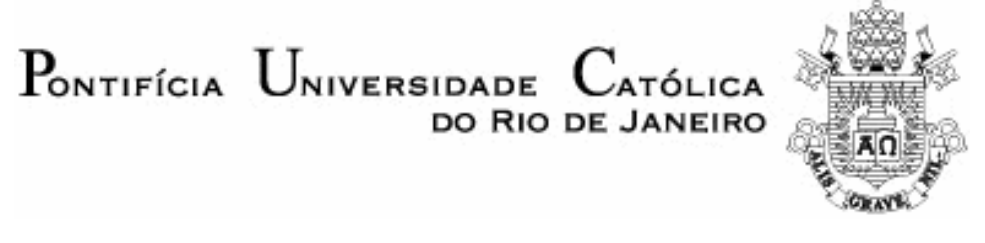

Melchisedeck Feitosa Correia

Visualização da solução do campo-livre do SASSI-2000

Dissertação de Mestrado

Dissertação apresentada como requisito parcial para obtenção do título de Mestre pelo Programa de PósGraduação em Engenharia Civil da PUC-Rio.

Orientador: João Luis Pascal Roehl

Rio de Janeiro, novembro de 2006 


\section{Visualização da solução do campo-livre do SASSI-2000}

Dissertação apresentada como requisito parcial para obtenção do título de Mestre pelo Programa de PósGraduação em Engenharia Civil da PUC-Rio. Aprovada pela Comissão Examinadora abaixo assinada.

João Luis Pascal Roehl

Orientador

DEC / PUC-Rio

Andréia Abreu Diniz de Almeida

DEC / PUC-Rio

Paulo Batista Gonçalves

DEC / PUC-Rio

Teresa Denyse P. Araújo

UFC

José Eugênio Leal

Coordenador(a) Setorial do Centro Técnico Científico - PUC-Rio

Rio de Janeiro, 27 de novembro de 2006 
Todos os direitos reservados. É proibida a reprodução total ou parcial do trabalho sem autorização da universidade, do autor e do orientador.

\section{Melchisedeck Feitosa Correia}

Graduou-se em engenharia civil pela Universidade Federal de Sergipe, UFS, em maio de 2004.

Ficha Catalográfica

Correia, Melchisedeck Feitosa
Visualização da solução do campo-livre do
SASSI-2000 / Melchisedeck Feitosa Correia ;
orientador: João Luis Pascal Roehl. - 2006.
105 f. : il. ; $30 \mathrm{~cm}$
Dissertação (mestrado em Engenharia
Civil)-Pontifícia Universidade Católica do Rio de
Janeiro, Rio de Janeiro, 2006.
Inclui bibliografia
1. Engenharia Civil - Teses. 2. Análise na
freqüência. 3. Campo-livre. 4. SASSI-2000. I. Roehl,
João Luis Pascal. II. Pontifícia Universidade Católica
do Rio de Janeiro. Departamento de Engenharia Civil.
III. Título.

CDD: 624 
Aos meus pais: Edelsio Santos Correia e Edna Feitosa Correia, pelos ensinamentos, compreensão e amor. 


\section{Agradecimentos}

A Deus por ter me concedido o dom da vida, inteligência e sabedoria;

Ao professor Roehl pela paciência, orientação acadêmica e pessoal;

A Paôla pelos esclarecimentos, sugestões e companheirismo;

Aos colegas de mestrado;

À Capes pelo apoio financeiro;

Aos meus amigos da Igreja Assembléia de Deus em Jacarepaguá pelo apoio espiritual, hospitalidade e honra;

Aos meus pais, Edelsio e Edna, aos meus irmãos, Ana Leia, Junior, Danielle e Marília, e à Cícera pelo estímulo, compreensão e amor imensurável, obrigado por serem minha família; 


\section{Resumo}

Correia, Melchisedeck Feitosa. Visualização da solução do campo-livre do SASSI-2000. Rio de Janeiro, 2006. 105p. Dissertação de Mestrado Departamento de Engenharia Civil, Pontifícia Universidade Católica do Rio de Janeiro.

Estudam-se as formas de resolução do campo-livre pelo programa SASSI2000 na análise de problemas de interação solo-estrutura, no domínio da frequiência, no caso de uma excitação sísmica. Considera-se um modelo de sítio composto por seis camadas de terreno, semi-infinitas, horizontais, apoiadas sobre um semi-espaço rígido e de uma estrutura aporticada, 3D, inserida no terreno, variando-se o nível de enterramento. Usam-se diferentes composições do campo-livre no que tange à natureza e ao ângulo de incidência das ondas que o compõem. Analisa-se, inicialmente, o comportamento do sistema no campolivre através dos parâmetros: eleição adequada dos tipos de onda, de sua inclinação com a vertical do ponto de controle e participações relativas na composição do sistema. Focalizam-se as variações das freqüências naturais, fornecidas por funções de transferência obtidas na vertical do ponto de controle, e dos respectivos modos de vibração. Repete-se a análise com a presença da estrutura variando-se o nível de enterramento da mesma e acrescentando-se, neste caso, o interesse na variação da cinemática do campo-livre além dos limites do terreno escavado. Conclui-se, finalmente, sobre as atitudes de trabalho e as seleções mais adequadas dos parâmetros para a utilização corrente do programa.

\section{Palavras-chave}

SASSI-2000; campo-livre; análise na freqüência. 


\section{Abstract}

Correia, Melchisedeck Feitosa. Visualization of free-field solution in SASSI-2000. Rio de Janeiro, 2006. 105p. MSc.Dissertation Departamento de Engenharia Civil, Pontifícia Universidade Católica do Rio de Janeiro.

One studies on SASSI-2000 frequency domain resolution procedures to obtain the so called free-field response for a site under seismic excitation, consideration made to the soil structure interaction. A stratified site model is considered formed by a series of six semi-infinite horizontal layers with equal height resting on a rigid viscoelastic half-space; the structure model is a $3 \mathrm{D}$ isosymmetric orthogonal frame made of equal beam elements with variable soil embedment. Different free-field wave compositions, including body and surface waves, are used with variable incidence angle to the vertical Z-axis.

The behavior of the free-field solution is observed through the following parameters and selections: wave types, value of their incidence angle to the vertical of the control point on the half-space surface and their relative participation factors in the overall wave field; the structure to soil mass and stiffness ratios are kept constant. One focalizes the variation of the system natural frequency values, obtained from a series of suitably selected transfer functions on the control point vertical line, and their associated modal configurations. The run series is repeated with the structure presence under different soil embedment and the observation is extended to the soil kinematics variation outside the excavated soil.

Conclusions include the understanding of the main behavior aspects and orientation is given about the more convenient attitudes and selections to be taken.

\section{Keywords}

SASSI-2000; free-field; frequency analysis. 


\section{Sumário}

Romanos 15

Gregos 17

1 Introdução 18

1.1. Motivação 18

1.2. Contextualização 19

1.3. Objetivos 21

1.4. Organização do texto 21

2 Revisão bibliográfica 23

2.1. Análise no domínio da freqüência 23

2.1.1. Transformada discreta de Fourier 23

2.1.2. Função de transferência 28

2.2. Propagação de ondas elásticas 30

2.3. Excitação sísmica 32

3 Programa SASSI-2000 36

3.1. Descrição geral 36

3.2. Ambientação $\quad 39$

3.3. Esquema de solução com diagrama de bloco 41

4 O Campo-livre, seus cenários e protagonistas. 43

4.1. Geral 43

4.2. Cenários do campo-livre no ambiente SASSI-2000 44

4.2.1. O cenário matemático 44

4.2.1.1. Ondas $\mathrm{P}$ e SV inclinadas 44

4.2.1.2. Ondas $\mathrm{SH}$ inclinadas 46

4.2.1.3. Ondas de Rayleigh, $R$

4.2.1.4. Ondas de Love, L 48

4.2.2. Cenários físicos 49

4.3. Protagonistas e seus papéis nos cenários do campo-livre 49 
5 Definição da composição das características do campo-livre 58

5.1. Estratégia 58

5.2. Programa de ensaio 60

5.2.1. Ondas de corpo (P, SV e SH) 60

5.2.2. Ondas superficiais (R e L) 61

5.3. Apresentação e análise dos resultados 62

5.3.1. Ondas de corpo (P, SV e SH) 62

5.3.1.1. Relação entre os resultados apresentados pelas ondas P e SV 62

5.3.1.2. Onda SH 76

5.3.2. Ondas de superfície (Rayleigh e Love) 80

5.3.2.1. Ondas Rayleigh 80

5.3.2.2. Onda Love $\quad 84$

5.4. Conclusões parciais (terreno - campo-livre) 86

6 Análise da interação solo estrutura 88

6.1. Estratégia 88

6.2. Resultados da interação solo estrutura 90

6.3. Conclusões parciais (terreno + estrutura) 97

7 Encerramento 99

7.1. Conclusões finais 99

7.2. Sugestões para prosseguimento 101

$\begin{array}{ll}\text { 7.3. Sinopse para os usuários } & 101\end{array}$

8 Referências bibliográficas 104 


\section{Lista de figuras}

Figura 2.1 - Esquema de análise de um sistema nos domínios do tempo e da

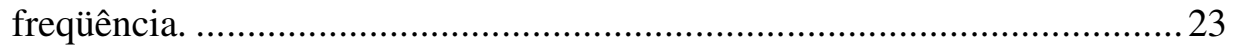

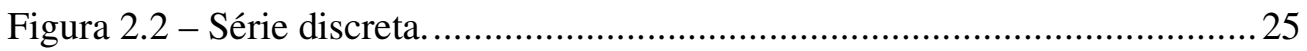

Figura 2.3 - Deformações produzidas pelas ondas de corpo: (a) ondas P; (b) ondas

SV. Fonte: W. H. Freeman and Company, apud Kramer 1996................... 34

Figura 2.4 - Deformações causadas pelas ondas superficiais: (a) ondas de Rayleigh; (b) ondas de Love. Fonte: W. H. Freeman and Company, apud

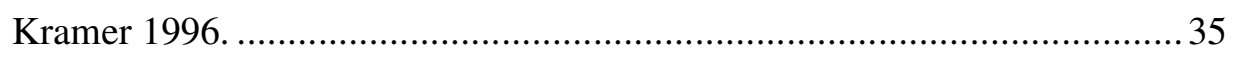

Figura 3.1 - Modelo de subestruturação do volume flexível. (a) Sistema Total; (b)

Sítio original, com indicação dos nós do volume de solo escavado e (c)

Estrutura. Fonte: manual teórico do SASSI-2000...................................... 37

Figura 3.2- Diagrama de bloco do SASSI-2000. Fonte: Manual do Usuário SASSI-2000. 42

Figura 4.1 - Modelo de onda SV plana incidente. Fonte: manual teórico do SASSI-2000. 45

Figura 4.2 - Modelo de onda plana SH incidente. Fonte: manual teórico do SASSI-2000. .46

Figura 4.3 - Graus de liberdade para ondas de Rayleigh. Fonte: manual teórico do SASSI-2000. 47

Figura 4.4 - Graus de liberdade ondas de Love. Fonte: manual teórico do SASSI2000 . .48

Figura 4.5 - Modelo simplificado para representação do terreno: estrutura trabalhando ao cortante. 53

Figura 4.6 - Modelo simplificado de uma camada. 56

Figura 4.7 - Funções de transferência para o sistema simplificado de uma camada.

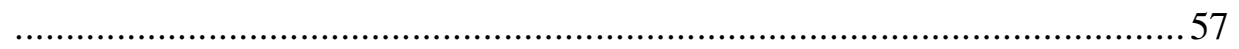

Figura 5.1 - Modelo da vertical do ponto de controle utilizado para as leituras ..58 Figura $5.2-1^{\circ}$ modo, vertical do ponto de controle, movimento de controle Z-X, solo 3. 65 
Figura $5.3-2^{\circ}$ modo, vertical do ponto de controle, movimento de controle $\mathrm{X}-\mathrm{Z}$, solo 3 .

Figura 5.4 - $3^{\circ}$ modo, vertical do ponto de controle, movimento de controle $\mathrm{Z}-\mathrm{X}$, solo 3 .

Figura 5.5- $4^{\circ}$ modo, vertical do ponto de controle, movimento de controle $\mathrm{X}-\mathrm{Z}$, solo 3 .

Figura 5.6 - $5^{\circ}$ modo, vertical do ponto de controle, movimento de controle Z-X, solo 3 .

Figura 5.7 - $6^{\circ}$ modo, vertical do ponto de controle, movimento de controle $\mathrm{X}-\mathrm{Z}$, solo 3 .

Figura 5.8 - Função de Transferência, movimento de controle Z-X, onda P, $\alpha=$ $0.5^{\circ}$, solo 3 . .70

Figura 5.9 - Função de Transferência, movimento de controle Z-X, onda P, $\alpha=$ $10^{\circ}$, solo 3 .

Figura 5.10 - Função de Transferência, movimento de controle Z-X, onda P, $\alpha=$ $20^{\circ}$, solo 3 .

Figura 5.11 - Função de Transferência, movimento de controle Z-X, onda P, $\alpha=$ $30^{\circ}$, solo 3 .

Figura 5.12 - Função de Transferência, movimento de controle Z-X, onda P, $\alpha=$ $35^{\circ}$, solo 3 .

Figura 5.13 - Função de Transferência, movimento de controle Z-X, onda SV, $\alpha=$ $0.5^{\mathrm{o}}$, solo 3 . 72

Figura 5.14 - Função de Transferência, movimento de controle Z-X, onda SV, $\alpha=$ $10^{\circ}$, solo 3 .

Figura 5.15 - Função de Transferência, movimento de controle Z-X, onda SV, $\alpha=$ $20^{\circ}$, solo 3 .

Figura 5.16 - Função de Transferência, movimento de controle Z-X, onda SV, $\alpha=$ $35^{\circ}$, solo 3 .

Figura 5.17 - Função de Transferência, propagação horizontal na superfície a cada $125 \mathrm{~m}$, movimento de controle $\mathrm{X}-\mathrm{Z}$, onda $\mathrm{P}, \alpha=35^{\circ}$, solo 3 . .74

Figura 5.18 - Função de Transferência, propagação horizontal na superfície a cada $125 \mathrm{~m}$, movimento de controle $\mathrm{Z}-\mathrm{X}$, onda $\mathrm{P}, \alpha=35^{\circ}$, solo 3 .

Figura 5.19 - Propagação horizontal com a freqüência a cada 125 m, movimento de controle $\mathrm{X}-\mathrm{X}$, onda $\mathrm{P}, \alpha=35^{\circ}$, solo 3 . 
Figura 5.20 - Propagação horizontal com a freqüência a cada 125 m, movimento de controle $X-Z$, onda $P, \alpha=35^{\circ}$, solo 3 .

Figura 5.21 - Função de transferência, movimento de controle Y-Y, onda SH, $\alpha=$ $0,5^{\circ}$, solo 3 .

Figura 5.22 - Funções de transferência, movimento de controle Y-Y, onda SH, $\alpha=$ $0,5^{\circ}$, solo 3 .

Figura 5.23 - Funções de transferência, movimento de controle Y-Y, onda SH, $\alpha$ $=35^{\circ}$, solo 3 .

Figura 5.24 - Funções de transferência, movimento de controle X-X, onda SV, $\alpha$ $=0,5^{\circ}$, solo 3 .

Figura 5.25 - Modos, vertical do ponto de controle, movimento de controle Y-Y, onda $\mathrm{SH}, \alpha=0,5^{\circ}$, solo 3 . 79

Figura 5.26- Função de Transferência, movimento de controle X-Z, onda R, solo 3.

Figura 5.27 - Função de Transferência, movimento de controle Z-X, onda R, solo 3.

Figura 5.28 - Função de Transferência, movimento de controle X-X, onda R, solo 3. 82

Figura 5.29 - Função de Transferência, movimento de controle X-X, onda R, solo 3.

Figura 5.30 - Modo fundamental, vertical do ponto de controle, movimento de controle Z-X e X-Z, ondas R e P, solo 3.

Figura 5.31 - Função de Transferência, movimento de controle Y-Y, onda R, solo 3.

Figura 6.1 - Modelo da estrutura enterrada até a $3^{\mathrm{a}}$ camada do solo e os pontos de leitura. 89

Figura 6.2 - Modelo da estrutura no SAP2000

Figura 6.3 - Os seis primeiros modos da estrutura e os modos correspondentes às frequiências de $25,20 \mathrm{~Hz}\left(9^{\circ}\right)$ e $83,77 \mathrm{~Hz}\left(43^{\circ}\right)$.

Figura $6.4-1^{\circ}$ Modo, vertical do ponto de controle, variação do nível de enterramento, movimento de controle $\mathrm{Z}-\mathrm{X}$, onda $\mathrm{SV}, \alpha=20^{\circ}$, solo 3 .

Figura $6.5-2^{\circ}$ Modo, vertical do ponto de controle, variação do nível de enterramento, movimento de controle X-Z, onda SV, $\alpha=20^{\circ}$, solo 3 . .93 
Figura 6.6 - $3^{\circ}$ Modo, vertical do ponto de controle, variação do nível de enterramento, movimento de controle Z-X, onda SV, $\alpha=20^{\circ}$, solo 3......... 94

Figura 6.7 - $4^{\circ}$ Modo, vertical do ponto de controle, variação do nível de enterramento, movimento de controle $\mathrm{X}-\mathrm{Z}, 2^{\circ}$ modo, onda SV, $\alpha=20^{\circ}$, solo 3.

Figura 6.8 - Modos de vibração com a distância, variação do nível de enterramento, movimento de controle X-Z, onda SV, $\alpha=20^{\circ}$, solo 3 ......... 96

Figura 6.9 - Superfície do solo, variação com a distância para vários níveis de enterramento, movimento de controle X-Z e Z-Z, onda SV, $\alpha=20^{\circ}$, solo 3. 


\section{Lista de tabelas}

Tabela 4.1 - Características físicas do modelo ..................................................55

Tabela 4.2 - Freqüências de referência .............................................................55

Tabela 5.1 - Parâmetros do campo-livre no modelo de sítio ................................61

Tabela 5.2 - Frequiências do sistema para o solo 3 para $\alpha=0,5^{\circ} \ldots \ldots \ldots \ldots \ldots \ldots \ldots \ldots . . \ldots 2$

Tabela 5.3 - Freqüências do sistema para o solo 3 para $\alpha=10^{\circ}$.......................63

Tabela 5.4 - Frequiências do sistema para o solo 3 para $\alpha=35^{\circ}$........................ 64

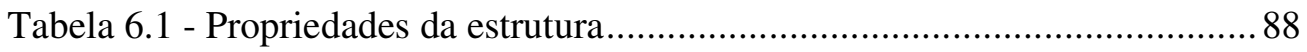

Tabela 6.2 - Freqüências da estrutura ....................................................... 90 


\section{Lista de símbolos e abreviaturas}

\section{Romanos}

a

$\mathrm{a}_{\mathrm{c} 1}$

A

NÓ i

S.E.Rig.

$\mathrm{EC} 1$

$\mathrm{EC} 2$

$\mathrm{EC} 3$

ESUP

CL

b

$b_{c}$

[C]

$\vec{e}$

$\mathrm{E}$

$f$

$F_{n}$

$f_{r}$

$f(t)$

$\mathrm{X}-\mathrm{X}$

$\mathrm{Z}-\mathrm{Z}$

$\mathrm{X}-\mathrm{Z}$

$\mathrm{Z}-\mathrm{X}$

$\mathrm{Y}-\mathrm{Y}$

$f_{o_{n}}$

G
Aceleração

Aceleração no topo da camada 1

Área

Nó no topo da camada i

Semi-espaço rígido

Estrutura enterrada até a primeira camada

Estrutura enterrada até a segunda camada

Estrutura enterrada até a terceira camada

Estrutura na superfície do solo

Campo Livre

Profundidade

Ângulo crítico de incidência da onda SV

Matriz de rigidez dinâmica complexa dependente da frequiência

Vetor unitário

Módulo de elasticidade

Frequiência em $\mathrm{Hz}$

Componentes da transformada discreta de Fourier de uma série

Componente $\mathrm{r}$ da série discreta

Função temporal

Movimento de controle na direção X, direção de análise X

Movimento de controle na direção Z, direção de análise Z

Movimento de controle na direção X, direção de análise Z

Movimento de controle na direção $Z$, direção de análise $X$

Movimento de controle na direção $\mathrm{Y}$, direção de análise $\mathrm{Y}$

Freqüência natural $n$

Módulo de elasticidade transversal 


\begin{tabular}{|c|c|}
\hline $\mathrm{h}$ & Espessura da camada de terreno \\
\hline$h(t-\tau)$ & Função resposta a um impulso unitário \\
\hline$H(\omega)$ & $\begin{array}{l}\text { Função de transferência (função resposta a um harmônico } \\
\text { unitário) }\end{array}$ \\
\hline $\bar{H}(\omega)_{i}$ & $\begin{array}{l}\text { Amplitude harmônica da resposta do modo } i \text { a um vetor } \\
\text { unitário }\end{array}$ \\
\hline I & Momento de inércia \\
\hline $\mathrm{J}$ & Momento torcional de inércia \\
\hline $\mathrm{k}$ & Número de onda \\
\hline$[\mathrm{K}]$ & Matriz de rigidez \\
\hline 1 & Largura \\
\hline $\mathrm{m}$ & Massa \\
\hline$[\mathrm{M}]$ & Matriz de massa total \\
\hline$M s$ & Massa de terreno por andar \\
\hline $\mathrm{N}$ & Número de pontos da transformada de Fourier \\
\hline $\mathrm{n}_{\mathrm{c}}$ & Número de camadas de terreno \\
\hline$N F$ & Número total de frequiências de análise \\
\hline NFreq $_{i}$ & Número da freqüência de análise i \\
\hline$\left\{P_{b}\right\}$ & Vetor de carregamento no topo do semi-espaço \\
\hline$r(\omega)$ & Resposta na freqüiência $\omega$ \\
\hline $\mathrm{S}$ & Área de cisalhamento \\
\hline$\tau$ & Transformada de Fourier \\
\hline$\tau^{-1}$ & Transformada inversa de Fourier \\
\hline $\mathrm{t}$ & Instante de tempo \\
\hline $\mathrm{T}$ & Período \\
\hline $\mathrm{TDF}$ & Transformada discreta de Fourier \\
\hline $\mathrm{TRF}$ & Transformada rápida de Fourier \\
\hline $\mathrm{u}$ & Deslocamento \\
\hline$\{U\}$ & Vetor de deslocamentos, na vertical do ponto de controle \\
\hline$\left\{U_{f}^{\prime}\right\}$ & Vetor de movimentos do campo-livre nos nós de interação \\
\hline$V$ & Velocidade da onda \\
\hline$V_{P}$ & Velocidade da onda P \\
\hline
\end{tabular}


$V_{S} \quad$ Velocidade da onda $\mathrm{S}$

$\{W\} \quad$ Modos de vibração

XPC, $\mathrm{y}_{\mathrm{PC}}$ Distâncias $\mathrm{x}$ e y do ponto de controle ao nó de interação

\section{Gregos}

$\alpha$

$\delta$

$\Delta f$

$\Delta t$

$\gamma$

$\eta$

$\lambda$

$\mu=v$

$\rho$

$\omega$

$\omega_{0 \mathrm{i}}$

$\xi_{\mathrm{P}}$

$\xi_{\mathrm{SV}}$
Ângulo de incidência das ondas

Fator de participação modal

Incremento de frequiência

Incremento de tempo

Massa específica

Fator de participação da onda de Rayleigh

Constante de Lamé

Coeficiente de Poisson

Peso específico

Freqüência circular

Frequiência circular natural i

Fator de amortecimento associado à onda $\mathrm{P}$

Fator de amortecimento associado à onda SV 